\title{
NON-TUBERCULOUS INFECTION OF THE URINARY TRACT
}

\author{
RalPh Shackman, M.B., B.S., F.R.C.S. \\ Consultant Surgeon and Urologist, Hammersmith Hospital
}

The diagnosis of non-tuberculous infection of the urinary tract is made by microscopic and bacteriological examinations of the urine: similar examinations of the urethral discharge are made when urethritis is present. Midstream specimens of urine, after careful cleansing of the prepuce and external urinary meatus by an 0.05 per cent. water solution of chlorhexidine in 0.5 per cent. cetrimide, is adequate in males. In females, however, it is traditional to obtain catheter specimens although it has been suggested recently (Boshell and Sandford, 1958) that 'clean' freshly voided specimens compare favourably, from the bacteriological aspect, with those obtained by catheterization.

The presence of organisms in the urine in the absence of a significant number of pus cells, generally speaking, may be disregarded, for the normal urethra commonly contains saprophytic bacteria (Harkness, 1950): staphylococci; Str. faecalis, diptheroids, Bact. coli; Str. viridans, and bacilli of the Proteus group have all, at times, been found to be present without evidence of urethritis (Shackman and Messent, 1954). Nevertheless, it is pertinent to point out that 22 per cent. of patients with chronic pyelonephritis have bacteria but no pus cells in the urine (Jackson et al., 1958): quantitative bacterial cultures are recommended by these authors and bacterial counts of 100,000 per ml. of freshly voided urine are said to be significant. There is generally a gross excess of the normal 3-4 pus cells per high-power microscopic field when there is acute inflammatory disease in the urinary tract but pyuria may be conspicuously absent in cases of renal carbuncle, perinephric abscess, or closed pyonephrosis, when the source of the infection, temporarily or ' permanently,' lacks a direct communication with the urinary passages: it is well, however, to repeat the urinalysis whenever the clinical features refute single negative laboratory observations.

There can be little doubt that the family doctor rather than the consultant, sees and successfully treats, without requesting microscopic and bac- teriological examination of the urine, the majority of patients in their primary attack of acute urinary infection: the incidence of 'honeymoon cystitis' and coliform cystitis in the female is extremely high. It is perfectly reasonable to manage such patients in this way: indeed, urological clinics and pathology services would probably be overwhelmed if such was not the case. Urinalysis, although always desirable, is thus frequently omitted without apparent untoward effects. On the other hand, undue persistence or recurrent attacks of urinary tract infection requires urological opinion and full investigation: so also does a frank haematuria. In such cases there may well be predisposing causes which require surgical treatment.

\section{Urological Investigation of Patients with Persistent or Recurrent Urinary Tract Infection}

Apart from microscopic and bacteriological examinations of the urine, good-quality $\mathrm{X}$-rays are required: a straight $\mathrm{X}$-ray of the whole of the urinary tract and, provided there is no sensitivity to iodine and the blood urea is not greater than $60 \mathrm{mg}$. per cent., excretion urograms should be carried out. In children, when the intravenous route is impracticable, the radio-opaque contrast medium, together with hyaluronidase, may be injected subcutaneously at the medial borders of the scapulae.

Cystoscopic examination is usually required but should be avoided in male patients who present with the clinical features of an acute cystitis. Instrumentation, despite extreme care and experience on the part of the operator, is liable to produce an exacerbation of the infective process: when necessary, cystoscopic examination is better carried out when the patient is an in-patient and under suitable chemotherapy or antibiotic cover. The risks of pyelonephritis and epididymitis are minimal when such precautions are taken.

Assessment of renal function is seldom indicated in patients who present with acute urinary tract 
infection: it is better to wait until the acute phase of the illness has subsided. Then the total glomerular function may be indicated by the blood urea, non-protein nitrogen, or creatinine concentrations, and the total renal tubule function by measurement of the urine specific gravity after 24 or 36 hours of fluid restriction (the fluid intake should be limited to $500 \mathrm{ml}$. during this time). In certain cases, for example patients who develop recurrent attacks of pyelonephritis after ureterointestinal anastomosis, or patients who have had chronic retention of urine, it is advisable to determine the serum electrolyte values: the minimal requirements are the serum bicarbonate and the serum potassium. In similar circumstances the haemoglobin estimation may be of value for an otherwise unexplained anaemia may be indicative of significant chronic renal disease.

The clinical examination of the patient should include the blood pressure, an examination of the external genitalia (including the penile urethra and the scrotal contents), a rectal examination with palpation of the prostate and seminal vesicles, and, when possible, a vaginal examination to exclude gross gynaecological abnormalities.

Other urological investigations which are sometimes indicated include ureteric catheterization, retrograde pyelography, retrograde cystography, and aortography, but they are seldom or never required in the acute phase of urinary tract infection.

\section{Pyelitis and Pyelonephritis}

It is doubtful whether pyelitis, nominally inflammation of the renal pelvis, ever occurs alone without some involvement of the renal parenchyma, and it is probably more accurate to use the term pyelonephritis if only to emphasize the potential risk of subsequent functional renal damage. The infection is usually haematogenous (Mallory et al., 1940) although ascent of organisms along the lumen of the ureter or their migration through the peri-ureteric lymphatics cannot be excluded entirely: suppurative pyelonephritis is a recognized serious complication of uretero-colic anastomosis and following instrumentation in cases of chronic retention of urine due to prostatic obstruction and it is difficult to refute the possibility of ascending infection in such cases.

Acute pyelonephritis may develop in patients of any age. It is seen in young children, in childbearing women, and in adult or old men. The condition is more common in females and the infection is thought to arise from the perineum: there is, however, no proof that the bacterial spread takes place along the urethra, although the short, straight female urethra would appear to predispose to this possibility. Congenital ab- normalities such as hydronephrosis, ureteri迎 stenosis, ureterocele, ectopia, or urethral valves predispose to pyelonephritis and are relativel more common in male children: urinary obstruc $\epsilon$ tion, horseshoe kidney, diabetes, neurogenic ab normalities, and instrumentation are other recog nized predisposing causes.

The diagnosis of acute pyelonephritis in childrere may be difficult: sometimes there is little or no reference to the urinary tract and fever, rigors, or an attack of screaming are the only clinical mani-s festations and acute appendicitis may be closely mimicked. Acute pyelonephritis of pregnancy, a not uncommon complication, occurs usually about $\vec{\omega}$ the fourth month and is more common on the right side. High fever, often with rigors, loin paino and local lumbar tenderness, increased urinary?. frequency, and dysuria, suggest the correct diag-w nosis but the clinical features of acute appendicitis, $\rightarrow$ torsion of an ovarian cyst, or necrobiosis of a ureteric fibroid are, at times, all too similar. Acute $\vec{n}$ pyelonephritis in adult males is usually secondary ${ }_{0}^{G}$ to a primary abnormality such as calculous, pelvi-근 ureteric obstruction, or prostatic obstruction, and $\vec{z}$ may progress to a pyonephrosis: the clinical mani- o festations are seldom obscure but loin tenderness may be conspicuously absent despite a severe $\overrightarrow{-}$ degree of renal parenchymal infection.

Treatment of acute pyelonephritis includes. strict bed rest, a low-protein high-carbohydrato diet, and excess bland fluids: the fluid intake should be enough to produce a daily urine output of at least 31 . Potassium citrate with hyoscyamus (National Formulary) should be prescribed and it $\stackrel{\square}{\varrho}$ is reasonable to give a sulpha preparation (vide infra) $\overrightarrow{\overrightarrow{0}}$ while the bacteriology report on the urine is $\frac{0}{3}$ awaited. The organisms are frequently gram negative bacilli and, especially in the uncomplicated case, the response to such treatment is rapid: in any case, little is lost even when the 3 . infection subsequently proves to be sulpha-re- $\frac{\bar{\sigma}}{3}$ sistant. Definitive drug therapy is determined only after full bacteriological sensitivity tests and $\delta$ complete urological investigation: remedial or $₹$ ablative surgery may be required.

Acute pyelonephritis generally undergoes resti- $D$ tution and subsequent renal function is ap- 을. parently normal: recurrent attacks, however, $N$ carry a significant risk of permanent renal damage. At times, the infection persists as a chronic lesion $\tilde{D}$ and may be overlooked until renal failure or hyper- N tension become manifest. Only rarely does death follow from sepsis and renal failure early in the disease.

Chronic pyelonephritis frequently differs in $\stackrel{\mathbb{D}}{\rightarrow}$ degree in the two kidneys and in different parts of $T$ a single kidney: the disease mainly affects the $\stackrel{\vec{D}}{\vec{D}}$ renal tubules which become dilated, filled with 


\section{Practical Obstetric Problems}

(2nd edition) by IAN DONALD

M.B.E., M.D.(Lond.), F.R.F.P.S.(Glas.), F.R.C.O.G.

Regius Professor of Midwifery, University of Glasgow

$x v i+712 p p$.

139 illustrations

(1959) 55s. net

viii $+200 p p$.

76 illustrations,

2 colour plates

(1958) 30s. net

$x v i+932 p p$.

410 illustrations,

3 colour plates

(1958) 84s. net

\section{Fluid Balance in Surgical Practice (2nd edition) \\ by L. P. LE QUESNE}

D.M.(Oxon), F.R.C.S.(Eng.)

Assistant Director, Department of Surgical Studies, Middlesex Hospital

viii $+140 p p$.

42 illustrations

(1957) 20s. net

\section{Newman Street, London, W.I}

thyroid-like colloid casts, cystic, or atrophic. Glomerular crowding results from disappearance of tubules. Plasma cells and other chronic inflammatory cells permeate the renal parenchyma and there is deposition of fibrous scar tissue. Such pathological changes, if extensive, are associated with significant diminution of renal function and give rise to azotemia and acidosis: abnormal sodium and potassium loss may also occur. Secondary hyperparathyroidism and renal dwarfism may develop.

Except during acute exacerbations, loin pain and tenderness are frequently absent in chronic pyelonephritis and the increased urinary frequency, accompanied by increased thirst, may be a reflection of inability of the renal tubules to conserve water. General malaise and an iron-resistant anaemia may occur. A low-serum bicarbonate, frequently associated with hyperchloremia, is a biochemical manifestation of functional tubule deficiency: the production of ammonia and the exchange of hydrogen ions for sodium-important normal functions of the renal tubule epithelium which help to maintain the body acid/base balance-are impaired and an abnormal urinary loss of base ions occurs. Calcium may be excreted in excess and nephro-calcinosis and multiple calculi may develop. When an abnormal loss of sodium occurs, the so-called ' salt losing nephritis' (Enticknap, 1952), dehydration, leg cramps, mental confusion, hypotension, and even Addisonian pigmentation (probably the result of a compensatory increased production of ACTH) may develop while an excessive potassium loss may give rise to a flaccid paresis similar to that produced by an aldosterone tumour of the adrenals.

Hypertension may be a sinister sequel to chronic pyelonephritis, not only when the kidney disease is bilateral-and here the treatment is clearly not surgical-but also when it is limited to a single kidney. Provided there are no irreversible hypertensive changes in the contralateral kidney, nephrectomy will cure the hypertensive disease. Unilateral chronic pyelonephritis may be suspected when a kidney, seen on a good-quality straight $\mathrm{X}$-ray, is abnormally small: thinning and irregularity of the cortex, demonstrable by excretion urography or retrograde pyelography is confirmatory. A decreased blood flow through such a kidney may be demonstrated by aortography or determined by divided renal function tests (Graber and Shackman, 1956). Divided renal function tests involve ureteric catheterization and measurements of para-amino hippurate or diodone clearances and are laborious. Alternative tests (Connor et al., 1957), if confirmed, might be 
preferable: a unilateral decreased urine flow with a low sodium concentration is said to be significant when renal arterial obstruction is present while an abnormally high sodium concentration is found when significant tubule damage exists. Renal biopsy by needle puncture may be advised but the patchy nature of the lesion limits the clinical value of this technique: a negative finding does not exclude disease, for the renal parenchyma may be perfectly normal at the site of the biopsy while active disease may exist only a centimetre away.

Treatment of chronic pyelonephritis is difficult: mechanical abnormalities associated with urine stasis, for example calculous, hydronephrosis, and bladder neck obstruction, should be treated surgically when possible but there can be no guarantee that pathological changes in the renal parenchyma will regress inevitably as a result of such treatment. Acute exacerbations of the disease should be treated by strict bed rest, an adequate fluid intake, a low-protein high-carbohydrate diet, and appropriate chemotherapy or antibiotic cover. Alkalis should be given, particularly when acidosis is present, and it is well to anticipate hypokalemia which may follow a. prolonged diuresis in an anorexic patient on a low potassium intake. Hypokalemia aggravates the anorexia by producing mental depression: it also has a direct adverse effect on the function of the cells of the renal tubules. Potassium administration, preferably by mouth, should be controlled by the measurement of the daily urine potassium output. It is possible that continued dosage of a suitable chemotherapeutic agent, by limiting spread of infection in the renal parenchyma, will prove to be of help in patients with chronic pyelonephritis: there is, however, no available literature on this aspect of the problem and personal experience is too small to quote.

When overt renal failure supervenes, death should not be expedited by over-enthusiastic fluid and electrolyte therapy. A strict fluid and electrolyte balance is required and biochemical analysis of the daily urine output is the key to replacement therapy: treatment should be based on sound physiology.

\section{Cystitis}

Patients with cystitis who are referred for specialist opinion have usually suffered more than a single attack and it is expedient, therefore, always to carry out urological investigations to confirm or refute the presence of a predisposing cause. In females, acute cystitis is not uncommon as a primary lesion but may be associated with urethral narrowing: it may also follow pelvic irradiation. In males, the condition is almost always secondary to prostatic or bladder neck obstruction, diverticu- lum, calculus, prostatitis, neurogenic dysfunction, or tumour: cystostomy and, rarely, vesico- $\frac{\varrho}{z}$ colic fistula may also be responsible.

Cystoscopy should be avoided, especially in ${ }_{c}$ male patients, in the acute phase of the disease: urinalysis and excretion urography are usually adequate primary diagnostic measures. The rareo condition known as Hunner's ulcer, a localized subepithelial and sometimes muscular fibrosis with $\frac{\bar{\rho}}{\bar{C}}$ chronic inflammatory reaction is an exception to $\stackrel{\mathbb{\complement}}{\varrho}$ this general rule for the instrumentation, particularly when the bladder is over-distended under anaesthesia, preferably extra-dural, seems to offer? some relief and, in any case, cystoscopy is the only $\overrightarrow{\vec{\omega}}$ way to make the diagnosis: the urine is charac- $\stackrel{\circ}{\circ}$ teristically sterile.

The symptoms of cystitis are often distressing and urinary frequency is accompanied by dysuria: $\omega$ sleep is disturbed. The degree of inflammation varies and may be intense enough to cause frank haematuria and mucosal sloughing.

Treatment consists of bed rest, adequate bland $N$

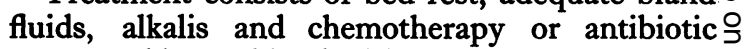
cover, and is combined with a search for an under- lying cause which may require surgical treatment. When there is alkali incrustation, an indwelling Foley urethral catheter and repeated bladder $?$ lavage with a solution of I per cent. Milton $\& \vec{G}$ often beneficial.

\section{Choice of Drugs}

The emergence of antibiotic-resistant staphylococci as a result of the universal and often haphazard administration of antibiotics is now well $\stackrel{\circ}{\propto}$ recognized: it is a problem of the greatest mag- $\overrightarrow{\vec{P}}$ nitude. It is perhaps not so well recognized that a $\frac{0}{3}$ somewhat similar situation has developed in the case of infections in the urinary tract. Organisms previously sensitive to the sulpha drugs and streptomycin are now frequently found to be $\frac{3}{3}$. resistant and moreover, as a result of selection, $\dot{\sigma}$ there is an increasing incidence of Proteus and 3 . $P s$. pyocyanea infections in our patients: paracolon $\delta$ bacilli and Bact. aerogenes are also more common. Dutton and Ralston (1957) have shown that patients admitted to hospital with Esch. coli infections which were sensitive to chemotherapy and antibiotic treatment contrasted strongly with those patients who acquired urological infection in . hospital. Urinary infections acquired outside the $N$ hospital environment are likely to be drug-sensitive and differ from infections acquired within the hospital and which are likely to be drug-resistant. Sensitivity tests should always be requested in the $\frac{C}{\Phi}$ latter case before definitive chemotherapy or anti- $\stackrel{f}{?}$ biotic treatment is begun but it might be reason- $T$ able to omit this examination in patients who have not been subjected, immediately or remotely, to 
instrumentation or operation, or in patients who have not suffered chronic or recurrent attacks.

Garrod et al. (1954) surveyed the efficacy of some chemotherapeutic and antibiotic agents in patients with urinary tract infections and reported immediate cure rates (judged five days after completion of the treatment) of 85 per cent., 82 per cent., and $8 \mathrm{I}$ per cent. respectively with trisulphonamide, sulphadimidine, and penicillin. It was noted, however, that these cure rates fell to 34 per cent. in males and $5^{8}$ per cent. in females when there was a demonstrable abnormalityapart from the infection-in the urinary tract, and that diminished cure rates also occurred when there was a previous history of urinary infection without urinary tract abnormalities.

Trisulphonamide is said to carry a minimal risk of crystalluria but sensitivity may develop after absorption of any of the soluble sulphonamides and an adequate fluid intake and alkali therapy should always be maintained throughout the period of their administration: the development of haematuria, loin pain, or oliguria should be regarded with suspicion. The low-dose, longacting sulphonamide preparations, such as sulphamethoxypyridazine, for use both in adults and children, are welcome but, apart from their convenience, do not differ in their mode of action from other sulpha preparations.

Nitrofurantoin is valuable as a broad spectrum antibacterial agent and is said to be effective against many strains of Bact. coli; some have claimed its value in some Proteus and some Str. faecalis infections. Its action is inhibited when the urine $\mathrm{pH}$ is 8.1 and optimal when it is 5.4. Mutant bacterial species resistant to nitrofurantoin are, however, now appearing, and organisms such as Ps. pyocyanea are becoming more common: parenteral Polymyxin B is the only effective treatment for Ps. pyocyanea infections and this antibiotic has earned a reputation of nephrotoxity.

Chloramphenicol remains the best antibiotic generally available for the treatment of urinary infections although chloramphenicol-resistant organisms may be found and there is a risk of aplasia of bone marrow following its use: the risk is minimal when treatment is limited to seven days. Chloramphenicol should never be given alone: it should always be given with tetracyclin, streptomycin, or a sulpha drug. In this way there is a minimal risk of development of resistant strains.

The sulphonamides, chloramphenicol, tetracyclin, and streptomycin effect their anti-bacterial activity in the blood stream and in the urine: the blood levels are great enough to be bacteristatic or bactericidal. They are, therefore, particularly valuable, in theory at least, in cases of pyelonephritis. By contrast, drugs such as nitrofurantoin, mandelic acid, methenamine mandelate, and the azo-dyes, act only in the urine: and since the blood levels are not great enough to inhibit or kill bacteria when the organisms are located deep in the tissues of the urinary tract it follows that a recurrence of urinary infection is liable to develop shortly after completion of a course of treatment with these particular drugs.

\section{Cross Infection in Urological Patients}

The risk of cross infection in urological patients justifies comment. There is little doubt that the risk is greatest in prostatic and bladder cases treated by suprapubic, retropubic, or transurethral methods, and when a urethral catheter or suprapubic tube remains in situ for any length of time: in such cases the urine may be sterile on admission to hospital but the patients are discharged home with infection in the urine. Although significant clinical effects may not be produced by this type of infection and the urine may become sterile again after a few months, the complication must not be disregarded and every attempt should be made to prevent it: on occasion, it may lead to serious secondary haemorrhage, pyelonephritis and septicaemia (Miller et al., I958).

Detailed bacteriological investigation in a male urological ward at Hammersmith Hospital by Dutton and Ralston (r957) has shown that the ward dust and air are a permanent reservoir of bacterial species which infect the urinary tract and that some degree of contamination of the nurses' hands is more or less inevitable. The risks of cross infection are, therefore, significant during catheterization, reconnection of a drainage tube after detachment from a catheter or bottle, or when sterilization and handling of the drainage bottles or urinals are imperfect. Bladder irrigations are particularly dangerous and it is expedient to avoid them both before and after operation unless there is significant bleeding and a risk of clot retention: a solution of I per cent. Milton is the only fluid used in our service for this purpose. Whenever possible, open-drainage should be avoided and drainage bottles, after adequate sterilization, should have cotton-wool bacterial filters in their air outlets. A closed-drainage system should be used not only in the prostatic and bladder cases but also, whenever possible, after surgery on the kidney and ureter.

Tactful education of the nursing staff and firm instruction to the junior medical staff have their place: the ritual of post-operative inspection of wounds should be minimal and conducted only with a strict anti-cross infection routine. To turn down the bed clothes and immediately expose a wound to the air-borne bacteria liberated from the bed linen is morally, and possibly legally, wrong. 
Now that there is an increasing proportion of hospital strains of bacteria resistant to the antibiotics it is paradoxical to take maximal care in the operating theatre and to disregard the risks incurred by imperfections in ward techniques.

\section{BIBLIOGRAPHY}

BOSHELL, B. R., and SANDFORD, J. P. (1958), Ann. intern. Med., 48, 1040 .

CONNOR, T. B., BERTHRONG, M., THOMAS, W. C., and

HOWARD, J. E. (1957), Bull. Yohns Hopk. Hosp., 100, 24I. DUTTON, A. A. C., and RALSTON, M. (1957), Lancet, i, I15.
ENTICKNAP, J. B. (1952), Lancet, ii, 458.

GARROD, L. P., SHOOTER, R. A., and CURWEN, M. R (1954), Brit. med. Э., II, 1003.

GRABER, I. G., and SHACKMAN, R. (1956), Brit. med. F., i, $1 3 2 \longdiv { \mathrm { D } }$

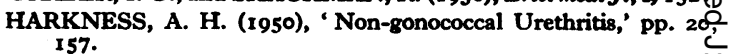
JACKSON, G. G., GRIEBLE, H. G., and KNUDSEN, K.
(1958), $\%$. Amer. med. Ass., 166, 14.

MALLORY, G. K., CRANE, A. R., and EDWARDS, J. E. (1940) Arch. Path. (Chicago), 30, 330.

MILLER, A., GILLESPIE, W. A., LINTON, K. B., SLADE, Niक and MITCHELL, J. P. (1958), Lancet, il, 608 .

SHACKMAN, R., and MESSENT, D. (1954), Brit. med. F., ip

\section{Amaremia of Pregmaney}

It has been generally accepted that incidence of megaloblastic anaemia of pregnancy in this country is very low. Reports indicate, however, that this type of anaemia may occur more frequently than has been suspected hitherto, but remains undetected in many cases. These observations suggest that it may be advisable to administer folic acid to all women in the last trimester of pregnancy.

Marmite yeast extract is a source of all known members of the vitamin B complex and has been used with conspicuous success in the treatment of nutritional megaloblastic anaemia of pregnancy. Its haemopoietic potency may be associated with the folic or folinic acid fraction. Marmite is readily incorporated in the diet and its pleasant taste ensures easy administration.

\section{MARMITE \\ yeast extract}

contains RIBOFLAVIN (vitomin $B_{2}$ ) $1.5 \mathrm{mg}$. per oz. NIACIN (nicotinic acid) $16.5 \mathrm{mg}$. per oz.

MARMITE LIMITED, WALSINGHAM HOUSE, SEETHING LANE, LONDON, E.C.30

\section{NOTICE OF SPECIAL INTEREST TO SUBSCRIBERS:}

'WHY NOT HAVE YOUR COPIES OF THIS JOURNAL BOUND INTO YEARLY VOLUMES?'

You can have your twelve monthly issues fully bound in dark green pin head doth. lettered in zilt on spine with name of Journal, Volume Number and year, complete with index at front, for 22s. 6d. post free. A limited number of out of print lournals are available to bind into volumes and make your library complete. Price on application giving details of issues required to complete back volumes.

THE FELLOWSHIP OF POSTGRADUATE MEDICINE GO PORTLAND PLACE, LONDON, W.I
WHY NOT

HAVE YOUR

JOURNALS

BOUND? 